Research Article

\title{
Exploring the Adoption of Nike+ Run Club App: An Application of the Theory of Reasoned Action
}

\author{
Chih-Wei Lin, ${ }^{1}$ Tso-Yen Mao, ${ }^{1}$ Ya-Chiu Huang, ${ }^{1}$ Wei Yeng Sia, ${ }^{2}$ and Chin-Cheng Yang $\mathbb{D I}^{1}$ \\ ${ }^{1}$ Department of Leisure Services of Management, Chaoyang University of Technology, 168 Jifeng E. Rd., Wufeng District, \\ Taichung 413, Taiwan \\ ${ }^{2}$ Department of Business Administration, Chaoyang University of Technology, 168 Jifeng E. Rd., Wufeng District, \\ Taichung 413, Taiwan \\ Correspondence should be addressed to Chin-Cheng Yang; ccyang.author@gmail.com
}

Received 8 January 2020; Accepted 6 May 2020; Published 23 June 2020

Guest Editor: Praveen Agarwal

Copyright () 2020 Chih-Wei Lin et al. This is an open access article distributed under the Creative Commons Attribution License, which permits unrestricted use, distribution, and reproduction in any medium, provided the original work is properly cited.

\begin{abstract}
The purpose of this study was to explore the influencing factors of users using Nike + Run Club App by Theory of Reasoned Action (TRA) as the theoretical basis and add the perceived playfulness into the research framework. This study took the users of the Nike + Run Club App as the research subject and distributed 360 questionnaires by snowballing sampling, a total of 351 valid questionnaires. All data were analyzed by descriptive statistics, confirmatory factor analysis, and structural equation models. Overall, the results reveal that extending TRA could be a well-explained users' behavior in the mobile application. The study found that the less the efforts spent in learning, the more positive the influence on attitude, thus affecting users' behavior. Therefore, this study proposed the following suggestions: People are pursuing a clearer and simple interactive function, a simplistic design; or adding instructions next to the new features will make the Nike + Run Club App more perfect. Emphasize the user's entertainment needs, develop interesting tasks, or games to make users feel interesting, and then be willing to continue to use the Nike + Run Club App.
\end{abstract}

\section{Introduction}

People in Taiwan use a mobile phone rather than a desktop or tablet. From the above information, we can know that, in Taiwan, the popularity of mobile devices is high, and it can be even said that they are a necessity of life. People have become accustomed to using mobile devices to do online work. The percentage of Taiwanese people using smartphones is increasing year by year; about $80 \%$ of users will carry their mobile phones with them. It can be seen that smartphones have become more and more relevant to people's lives. Smartphones have a rich user interface, functions, and complex operating platforms, and a variety of apps are available to users. With the popularity of smartphones and the maturity of mobile networks, people's lives are gradually changing, becoming more convenient.
Nowadays, people's awareness of health is improving. For example, running is the innate instinct of people, which is a leisure sport that is easy to engage where people are less burdened [1]. Chu and Cho pointed out that jogging is an economical and efficient sport that is not limited by time, space, and age [2]. Chen also believes that jogging has health benefits for people of all ages, and regular exercise promotes the quality of life and health of people [3]. In addition, the National Sports Awareness Survey found that the most popular sports in Taiwan were "jogging, brisk walking, and walking," accounting for $51.7 \%$ [4]. Obviously, jogging has gradually become the mainstream of sports and leisure, and it is not limited by time and space, making it the first choice for sports and leisure.

Along with the emergence of smartphones, many health managements related to mobile applications have been developed through mobile smart devices to download mobile applications. Sports help athletes track or record 
their individual sports status and promote the spirit of national sports for health or fitness purposes. App software companies also offer much different software for sportsloving users. The sports app is simple and easy to carry, and most of the sports apps have a wide range of functions and popular among sports and fitness enthusiasts. The sports app not only connects people and people, but also incorporates the concepts of smart health, medical care, and sports. It is tailor-made and records the most appropriate exercise habits and links the community to motivate each other and transform the movement into action community activities. According to foreign media reports, in recent years, the number of sports and fitness apps is growing at a rate of $150 \%$ per year. This showed that the population using mobile apps is growing and that sports apps are becoming more popular.

Since the mobile app is a medium for users to communicate with smartphone devices, it also drives the development of various types of apps, which not only creates a huge market for apps, but also enriches the additional functions of mobile devices. Many companies have also joined the ranks of creating exclusive brand apps, trying to open up more possibilities for the overall operation of the company through marketing. Based on this, Nike has created more links with consumers in the app market and has developed several apps related to its brand, which is designed for road runners. For example, Nike + Run Club App provides runners with calorie calculations, mileage, time, community links, music, GPS positioning, and more. The number of apps in the app stores exceeded 1.5 million in 2015 [5].

Sports economics has been rising rapidly globally; enterprises integrate technology to make playing sports smarter as sports applications became popular in lifestyle. Sports applications are built for people to stay exercising, but nowadays with the multiple functions of the applications, $\mathrm{Tu}$ et al. found that making apps fun and interesting could help consumers sustain their effort in physical activity [6]. However, there are a lot of apps on the market, and how to be popular among users is an important issue. Although there are several apps in the mobile app store, the Nike + Run Club, which is the most popular among runners, is quite in line with the needs of runners, including friends cheering, self-selected songs, and sports star voice response. Being designated, fun, and easy to use made Nike+Run Club become the preferred app for many runners. It can be seen that Nike + Run Club has a large number of users; with the accelerated of sport application, it has become the trend for global, and it can be seen that it is necessary to understand the adoption for sport application. Therefore, this study uses the Nike + Run Club App as the research topic to explore the influencing factors affecting users' use of the Nike + Run Club App and further analyze users' willingness to continue using the app.

How to effectively predict or explain whether users accept information technology is a topic of concern for enterprises and organizations. It is also one of the most mature areas of information management development today [7]. Theory of Reasoned Action (TRA) is a widely used model in the field of social psychology. The advantage is that the factors affecting action need to be determined by affecting behavioral attitudes, subjective norms, or both. TRA has been proven to be effective in predicting and interpreting practical actions in many fields, and it has been supported by many preliminary evaluation studies. Therefore, this study uses TRA as the theoretical basis to explore the behavioral intentions of Nike + Run Club App users.

With the advancement of science and technology, people's lives have begun to be closely related to these technological products. The demand for these technology products has gradually increased, and products that satisfy the user's senses and pleasures will attract consumers [8]. Lin et al. mentioned that consumers are willing to pay more attention to the function of technology products and expect products to bring out excellent experience and good quality [9]. The Nike + Run Club App is an interesting application. In the Nike + Run Club App, you can record your running time and mileage, and the data can know the pace of each minute. Besides that, you can check out friends and relatives' records to stimulate the user's active mentality and have fun in the process. In recent years, the rapid development of technology has made the "fun" element gradually attract the attention of the design field. Lin pointed out that pleasure will enhance the user's intention of IT products [10]. It can be seen that the making the app interesting has also become a new direction in mobile application design. Therefore, this study incorporates perceived playfulness into the factors of attitude influence to understand the behavior intentions of Nike + Run Club App users.

To explore the behavior intention of the Nike + Run Club App, the purpose of this study is summarized as follows: 1 . Exploring the influence of perceived usefulness, perceived ease of use, and perceived playfulness on attitude. 2. Exploring the influence of Internet word-of-mouth and interpersonal influence on subjective norms. 3. Exploring the influence of attitude and subjective norms on behavioral intentions. Finally, the results of the research can be provided as a reference for the relevant mobile application developers and mobile applications and can also be used as a reference for future research.

TRA was proposed by Flanders et al. in 1975. TRA believes that the individual's behavioral intentions will be influenced by the attitude of the individual and subjective normative, and the behavior intention further influences the specific behavior being manifested. In other words, TRA assumes that "behavior occurs based on the control of the individual's will" [11]. If a person thinks that this behavior should occur, this behavior will occur, mainly used to understand and predict personal behavior. Faith represents a person's will to control their behavior. The behavior is generated by a person's belief in logical thinking, after choosing the right or not, or other decisions to take. TRA assumption is not affected by the external environment; that is, the idea of support can represent a person's behavior.

Taylor and Todd pointed out that users' attitudes toward using information technology (IT) will also be affected by perceived usefulness and perceived ease of use [12]. When 
the user perceives that the system is useful, the attitude toward the system will be more positive. In other words, when users feel that the function of using this innovative technology will improve the efficiency of learning or work and they need to spend less effort in learning, it will produce positive reviews. Flanders et al. argue that attitude is the result of an individual's past learning experience, producing a preference or aversion to the consistency of an object. The attitude is also an individual's overall evaluation of a particular person, thing, or idea [11]. Davis defines the attitude of use as an individual's positive or negative perception of the technology. This study divides the factors affecting attitude belief into perceived usefulness, perceived ease of use, and perceived playfulness [13].

Perceived usefulness and perceived ease of use are the two main beliefs in technology acceptance model (TAM). In 1986, Davis developed the TAM behavioral model based on TRA. It is designed for users to accept new information systems. TAM's purpose is to find an effective behavioral model to explain the behavior of users in computer technology to accept new information systems and to analyze the factors that affect user acceptance. TAM provides a theoretical basis for understanding the impact of external factors on users' beliefs, attitudes, and intentions, thus affecting the use of technology, and can be widely used to explain or predict the influencing factors of IT use.

The TAM uses perceived usefulness and perceived ease of use as independent variables, and attitudes, behavioral intentions, and usage behaviors are dependent variables. Advocating usefulness and ease of use can affect the attitude of using technology, which in turn affects specific behavioral performance. It also advocates that people's use of information technology is affected by their behavioral intentions.

Davis et al. pointed out that when the user perception system is easy to use, it will encourage users to do more work and improve job performance. Potential users subjectively believe that using a particular information system will increase their job performance or the likelihood of learning performance [14]. Davis pointed out the extent to which potential users subjectively believe that the operation of a particular information system technology is easy to use [13]. While the mind of the user causes a burden, it will produce negative emotions and then exclude the use. That is, when the user perceives the operation of the information system function of the innovative smartphone, the less the effort needed to be spent in learning, the more positive the attitude of using the system, and the perceived ease of use will also positively affect the perceived usefulness.

Perceived playfulness is defined as the degree to which a person feels euphoric when participating in an activity or adopting a system [15]. Moon and Kim applied the TAM to the World Wide Web study, citing research by Lieberman and Barnett to develop the third variable "perceived playfulness" [16-18].

Playfulness was first proposed by Lieberman, and then Barnett studied human behavior and made some observations about the meaning of playfulness: 1 . He focused on the interesting features (trait of playfulness), which regards interest as a feature of motivation, mainly referred to the characteristics of individuals that are more stable and do not change with the situation. 2. He considered playfulness to be caused by the interaction between the individual and the situation. It is mainly that the individual is affected by things in the scriptures and will be affected by time, by context factors, and by interaction $[15,18]$.

According to Csikszentmihalyi, if a person feels pleasant when interacting with the environment, this feeling will generate perceived playfulness, and therefore people will have a positive attitude toward the environment [19]. Lu and $\mathrm{Ma}$ also pointed out that the main reason why the product found that it can trigger the user's "pleasure" feeling is that the product can trigger the user's "emotional" experience, and the area is no longer limited to the "function" of the product itself [8]. Lin et al. pointed out that, by enhancing the happy atmosphere, users can get a positive evaluation and thus improve their behavioral intentions [20].

According to Flanders et al., TRA refers to the social pressure exerted by others or groups (such as parents, spouses, friends, and colleagues) on an individual's specific behavior and is based on normative beliefs and motivation to comply [11]. The normative belief refers to the opinion of other people or groups on an individual's engagement in a particular behavior; motivation to comply refers to the degree of compliance of individuals with other people's opinions. Ajzen believes that individuals' behavior will be influenced by the degree of identity of others and the surrounding environment [21]. Subjective norms are individuals who are subject to the pressure of important others, groups, and society. The above individuals are obedient to the decision or behavior, and the individual will change the self-determination behavior because of external factors.

When potential adopters try to adapt, to reduce uncertainty, the experience of the previous adopter (interpersonal influence) or the experts of the mass media in the field (external influence) will be consulted in advance. The potential adopter is to form his perspective on innovation from two sources (interpersonal influence and external influence). Many studies have confirmed that reference groups have an impact on behavioral intentions [22-24]. According to Bhattacherjee, interpersonal influence is influenced by the dictation of friends, superiors, classmates, and other innovators [24].

Research on the influence of word-of-mouth has been flourished in recent years. In past research, scholars have a lot of discussions and definitions of word-of-mouth communication. Warrington defines word-of-mouth communication as to how consumers shape their attitudes and behaviors [25]. "Word-of-mouth" has always played a very important role in the communication of products and services between consumers through nonvendor marketing channels [26]. Word-of-mouth communication is often one of the main considerations when consumers are faced with purchasing decisions about goods or services [27]. Therefore, word-of-mouth is a noncommercial, two-way communication, experience-oriented, interactive, and immediate [25].

Behavior intention refers to the tendency and degree of action of an individual who wants to engage in a particular 
behavior, that is, the psychological strength of the individual's action in the decision-making process; in measurement, it can be transformed into whether the individual is willing to try or is willing to pay, by which variables can explain and predict the actual performance of the individual.

In summary, TRA holds that attitude and subjective norm determine the individual's behavior intention; behavior intention determines the individual behavior, and the behavior intention is determined by the attitude and subjective norms. Therefore, if an individual is a more positive attitude on a particular behavior and subjective norms support the behavior, the individual's intention to engage in the behavior will also increase.

\section{Materials and Methods}

2.1. Research Subject. This study took the Nike + Run Club App users in Taiwan as the research subject and used the Google form to distribute the survey by purposive and snowball sampling. In addition to posting questionnaires on the various jogging-related community website, the study also distributed questionnaires to users who use Nike + Run Club and requested them to send to each other. The questionnaires were distributed online from December 26th, 2016, to January 26th, 2017; a total of 360 questionnaires were collected, with 351 valid questionnaires; the effective recovery rate was $97.5 \%$.

2.2. Research Tools. The scale of this study could be divided into three parts. The first part was the TAM, which was mainly referred to by Davis, Su, and Liao [13, 28, 29]. Through the Confirmatory Factor Analysis (CFA), the factor loading of attitude was between 0.85 and 0.89 , Composite Reliability (CR) is 0.93, and Average Variance Extracted (AVE) is 0.76 ; the factor loading of perceived usefulness was between 0.88 and $0.93, \mathrm{CR}$ is 0.94 , and AVE is 0.80 ; the factor loading of perceived ease of use was between 0.84 and 0.90 , CR is 0.93 , and AVE is 0.75 .

The second part would be the TRA, which was mainly referred to by Flanders et al., Bhattacherjee and Chen et al. $[11,24,30]$, Through the CFA, the factor loading of interpersonal influence was between 0.84 and 0.90 , CR is 0.90 , and AVE is 0.76; the factor loading of Internet $\mathrm{W}-\mathrm{O}-\mathrm{M}$ was between 0.80 and $0.92, \mathrm{CR}$ is 0.93 , and AVE is 0.77 ; the factor loading of the subjective norm was between 0.70 and 0.91 , $\mathrm{CR}$ is 0.88 , and AVE is 0.71 ; the factor loading of behavior intention was between 0.64 and 0.92 , CR is 0.89 , and AVE is 0.68 .

Finally, perceived playfulness was mainly referred to by Moon and Kim, and Cheng et al. [16, 30]. Through the CFA, the factor loading of perceived playfulness was between 0.87 and $0.90, \mathrm{CR}$ is 0.93 , and AVE is 0.78 .

2.3. Discriminant Validity. This study used the confidence interval method to test the discriminant validity of the overall behavior model. According to Torkzadeh et al., in confidence interval method, if the confidence interval does not contain 1 representative, there is no correlation at all, indicating that the variables have discriminant validity [31]. In this study, the bootstrap method was used to estimate (2,000 times), the confidence level was below 95\%, and percentile confidence intervals and bias-corrected confidence intervals were used to measure. In this study, the upper bounds of the confidence interval method are between 0.50 and 92 , respectively, and the lower bounds are between 0.34 and 0.81 ; the confidence interval does not contain 1 , indicating that each variable has discriminant validity.

2.4. Data Analysis. In this study, we used SPSS version 21.0 and AMOS version 21.0 to analyze the data and used descriptive statistics, confirmatory factor analysis, and structural equation modeling to understand the issues and purpose discussed in this study.

\section{Results and Discussion}

3.1. Subject Data Analysis. The subject majority in this study were females (62.7\%), aged around 21-30 (55.8\%); education level of the majority was university degree (79.8\%); occupation of the majority was student (60.1\%); average monthly income was below NTD 20,000 (60.7\%).

\subsection{Confirmatory Factor Analysis of the Apple Watch User} Behavior Model. Data of this study were qualified with the normality test but the $\mathrm{CR}$ of multivariate kurtosis was 107.20, the assumption of multivariate normality distribution was not supported; therefore, this study used bootstrap proposed by Bollen and Stine to modify the overall model [32]. In the overall model fit analysis, all values have reached the standard, GFI $=0.95, \quad$ RMSEA $=0.04, \quad \mathrm{NFI}=0.95$, $\mathrm{RFI}=0.94, \mathrm{IFI}=0.98, \mathrm{TFI}=0.98, \mathrm{CFI}=0.98, \mathrm{PGFI}=0.87$, $\mathrm{PNFI}=0.87, \mathrm{CN}=0.231 .88$ and $\chi^{2} / \mathrm{d} f=1.516$, indicating that the overall model fits well, and then continue the discussion.

For behavior intention, the attitude has a higher influence (0.73), which means that using Nike + Run Club App is meaningful and helpful to the sport, and the higher the user's attitude is, the higher the behavior intention will be (Figure 1).

For attitude, the influence of perceived ease of use is the highest (0.56), which means that, on behalf of users, the Nike + Run Club App is easy to operate and easy to use. According to Cheong and Park, the less the effort for users to learn the new information technology, the more positive the attitude on IT [33]. Followed by perceived playfulness (0.51), when using the Nike + Run Club App, on behalf of the user, it is fun to enjoy while running through the Nike + Run Club App (Figure 1). According to Csikszentmihalyi, if the user feels happy when interacting with technology, perceived playfulness will generate, and therefore the user will have a positive attitude and thus willingness to continue using Nike + Run Club App in the future [19].

For subjective norm, interpersonal influence has the higher influence (0.56), which means that users believe that the opinion and suggestion from friends and family are important for them to use the Nike + Run Club App; users 


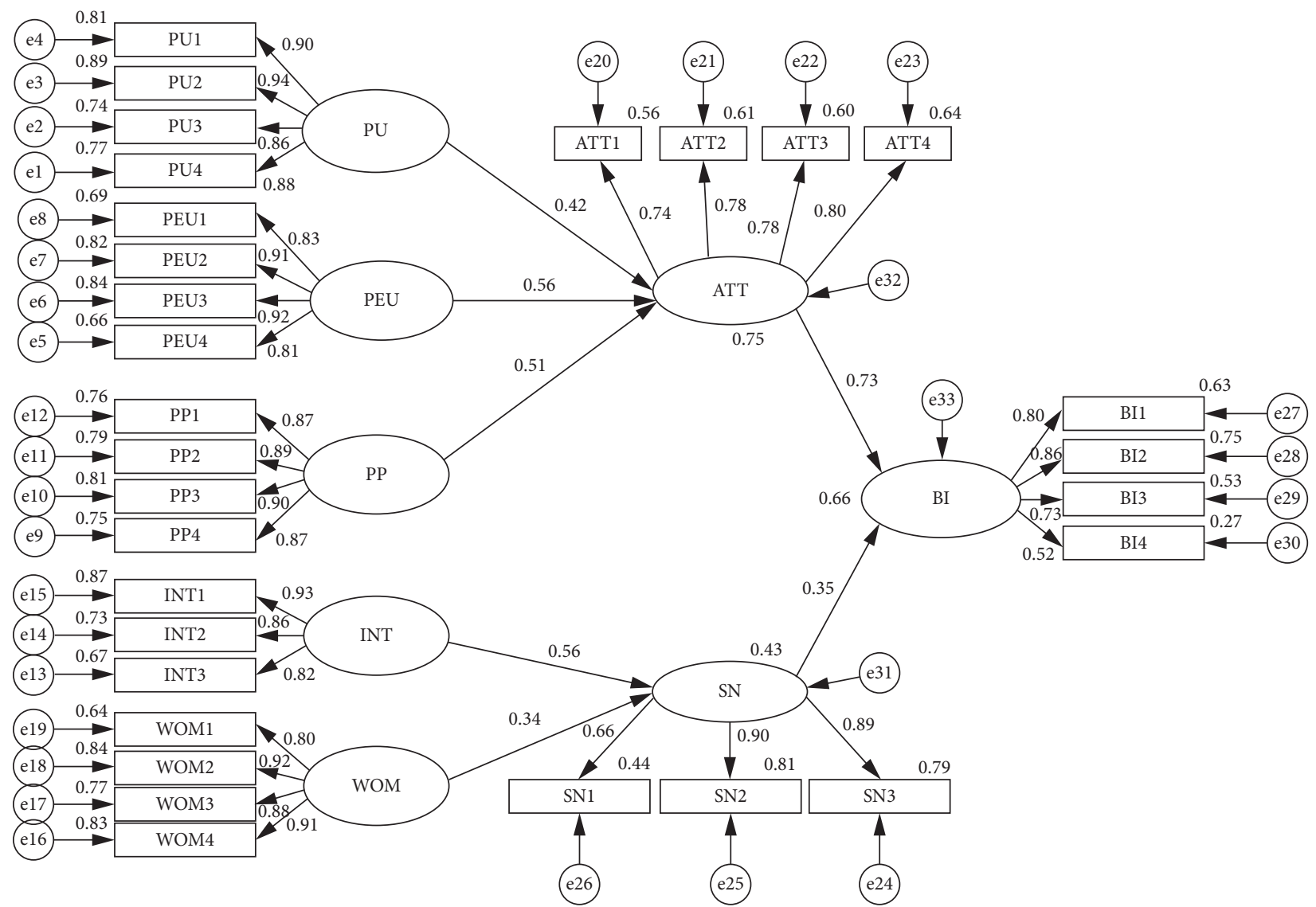

FIGURE 1: Path analysis of Nike Run + behavioral model. PU: perceived usefulness. PEU: perceived ease of use. PP: perceived playfulness. INT: interpersonal influence. WOM: internet word-of-mouth. ATT: altitude. SN: subjective norm. BI: behavior intention. e: error.

also hope that they can build a connection with people through the Nike+Run Club App (Figure 1). Therefore, when the interpersonal influence is more positive, the subjective norm will be higher. The results are similar to Lin's study results [34].

According to the results of this study, perceived ease of use has the greatest influence on attitudes; hence the designation of the Nike + Run Club App should be based on simple operations. The updated Nike + Run Club App is too complicated due to the new features. Although new interactive features have been added, there is no clear instruction of the operation, which leads users to update the Nike + Run Club App but cannot experience more diverse functions after the update. Therefore, this study suggests that, in a technologically advanced society, people are pursuing a clearer and simpler interactive function, so they can design in the direction of making the interactive function more simplistic or add instructions to the next function to make Nike + Run Club App More perfect.

According to the results of this study, the influence of perceived playfulness has a very high influence on attitude. When the questionnaire was further examined, it was found that users could enjoy the operation when using the Nike + Run Club App, but they could not satisfy their entertainment. The possible reason is that when jogging through the Nike+Run Club App, the user is interested because of the freshness, but if the user interacts for a long time, it lacks the incentive for the user to continue to feel the entertainment, leading to the lower entertainment demand. Therefore, this research suggests that, in addition to the user's refreshing features, it is also possible to develop interesting tasks or games into the Nike + Run Club App, emphasizing the user's entertainment needs and improving the Nike + Run Club App into a fun and enjoyable experience.

According to the results of this study, interpersonal influence has the greatest influence on the subjective norm, and the subjective norm will also influence behavior intention. It can be seen that family and friends will influence users to continue to use the Nike + Run Club App. Therefore, it is recommended that the Nike+Run Club App R\&D Department can be more closely connected to the community and users so that future users can continue to use the Nike + Run Club App trend.

This study used Nike Run + App as the major study research, but there are still a lot of applications on the market. Especially in a wearable device, Rause pointed out that wearable technology will be improving the exercise habits of adults [35]. Therefore, it is suggested that future research could study the application adoption of the wearable device.

This study distributes the survey on the jogging-related community website, yet we have found that the age of the 
majority of subjects was around $21-30$. Therefore, it is suggested that future research should distribute the survey through various paths to expand the sample to other age groups.

This study uses TRA as the theoretical basis to explore the behavioral intentions of Nike + Run Club App users. However, Lin et al. found that the adoption of application will be affected by the perceptual barrier, which has a significant negative impact on perceptual usefulness and perceived ease of use [36]. Therefore, it is suggested that future research should explore the adoption of application from the innovation resistance theory perspective.

\section{Conclusions}

Perceived usefulness, perceived ease of use, and perceived playfulness have positive and significant influence on attitude. Among them, perceived ease of use has the highest influence on attitude, indicating that users think that the operation of the Nike + Run Club App is the main factor affecting their attitude.

Interpersonal influence and Internet $\mathrm{W}-\mathrm{O}-\mathrm{M}$ had positive and significant influence on the subjective norm. Among them, interpersonal influence has the highest influence on the subjective norm, indicating that users believe that the recognition and suggestions of family and friends for the Nike + Run Club App are the main factors affecting subjective norm.

Attitude and subjective norm had positive and significant influence on behavior intention. Among them, the influence of attitude on behavior intention is the highest, indicating that the practicality of the Nike + Run Club App is an important factor affecting the continuous use of users.

\section{Data Availability}

The Nike Run + behavioral model data used to support the findings of this study are restricted by the Sports Research Center of Department of Leisure Services Management to protect the subject's privacy. Data are available from Dr. Chih-Wei Lin (e-mail: cwlin@cyut.edu.tw) for researchers who meet the criteria for access to confidential data.

\section{Conflicts of Interest}

The authors have no affiliations with or involvement in any organization or entity with any financial interest or nonfinancial interest in the subject matter or materials discussed in this manuscript.

\section{Acknowledgments}

The authors are thankful to the volunteers for their enthusiastic participation.

\section{References}

[1] C. Chen, "A study on flow experience and leisure benefits of road running participants," Journal of Physical Education Fu Jen Catholic University, vol. 10, pp. 230-244, 2011.

[2] S. L. Chu and S. Y. Cho, "Healthy exercise: start from jogging," Sport Research Review, vol. 72, pp. 192-199, 2004.

[3] K. Chen, "Jogging can increase survival and reduce disability for elder men," Health World, vol. 273, no. 9, 2008.

[4] Sport Administration, "Investigation and Research on the Consciousness of Taiwan People's Movement," 2013, https:// www.sa.gov.tw/Resource/Other/f1451381749304.pdfv.

[5] Kknews, "App service subscription is the future app store can no longer be confused," 2016, https://kknews.cc/zh-tw/tech/ le6lgzz.html.

[6] R. Tu, P. Hsieh, and W. Feng, "Walking for fun or for "likes"? the impacts of different gamification orientations of fitness apps on consumers' physical activities," Sport Management Review, vol. 22, no. 5, pp. 682-693, 2019.

[7] P. J. Hu, P. Y. K. Chau, O. R. L. Sheng, and K. Y. Tam, "Examining the technology acceptance model using physician acceptance of telemedicine technology," Journal of Management Information Systems, vol. 16, no. 2, pp. 91-112, 1999.

[8] C.-C. Lu and M.-Y. Ma, Research on the Attractive Factors of Pleasurable Products and Pleasure Durability, National Cheng Kung University, Tainan, Taiwan, 2010.

[9] C.-W. Lin, C.-C. Yang, W. Y. Sia, and K.-Y. Tang, "Examining the success factors of smart watch: a behavioral perspective on consumers," Polish Journal of Management Studies, vol. 20, no. 2, pp. 368-378, 2019.

[10] C.-W. Lin, T.-Y. Mao, W. Y. Sia, and W. P. Tan, "Extending the TAM to explore the behavior of user in using the innovative game console," in Basic \& Clinical Pharmacology \& Toxicology, Wiley, Hoboken, NJ USA, 2019.

[11] N. A. Flanders, M. Fishbein, and I. Ajzen, Belief, Attitude, Intention and Behaviour: An Introduction to Theory and Research, Addison-Wesley, Boston, MA, USA, 1975.

[12] S. Taylor and P. A. Todd, "Understanding information technology usage: a test of competing models," Information Systems Research, vol. 6, no. 2, pp. 144-176, 1995.

[13] F. D. Davis, "Perceived usefulness, perceived ease of use, and user acceptance of information technology," MIS Quarterly, vol. 13, no. 3, pp. 319-340, 1989.

[14] F. D. Davis, R. P. Bagozzi, and P. R. Warshaw, "User acceptance of computer technology: a comparison of two theoretical models," Management Science, vol. 35, no. 8, pp. 982-1003, 1989.

[15] P. Barnett, "Definition, design, and measurement," Play and Culture, vol. 3, pp. 319-336, 1990.

[16] J.-W. Moon and Y.-G. Kim, "Extending the TAM for a worldwide-web context," Information \& Management, vol. 38, no. 4, pp. 217-230, 2001.

[17] L. A. Barnett, "The playful child: measurement of a disposition to play," Play and Culture, vol. 4, no. 1, pp. 51-74, 1991.

[18] J. N. Lieberman, Playfulness: Its Relationship to Imagination and Creativity, Academic Press, Cambridge, MA, USA, 2014.

[19] M. Csikszentmihalyi, Beyond Boredom and Anxiety, JosseyBass, San Francisco, CA, US, 1975.

[20] Y.-S. Lin, Y.-C. Tseng, Y.-X. Kang, W. Y. Sia, and C.-W. Lin, "Wii sport's involvement in physical experience of national junior high students' physical education curriculum," in 
Proceedings of the 2nd International Conference on Education and E-Learning, Bali, Indonesia, November 2018.

[21] I. Ajzen, "The theory of planned behavior," Organizational Behavior and Human Decision Processes, vol. 50, no. 2, pp. 179-211, 1991.

[22] R. Agarwal and J. Prasad, "The role of innovation characteristics and perceived voluntariness in the acceptance of information technologies," Decision Sciences, vol. 28, no. 3, pp. 557-582, 1997.

[23] E. Rogers, Diffusion of Innovations, ACM, New York, NY, USA, 4th edition, 1995.

[24] A. Bhattacherjee, "Acceptance of e-commerce services: the case of electronic brokerages," IEEE Transactions on Systems, Man, and Cybernetics_Part A: Systems and Humans, vol. 30, no. 4, pp. 411-420, 2000.

[25] T. Warrington, "The secrets of word-of-mouth marketing: how to trigger exponential sales through runaway word of mouth," Journal of Consumer Marketing, vol. 19, no. 4, pp. 364-366, 2002.

[26] E. W. Anderson, "Customer satisfaction and word of mouth," Journal of Service Research, vol. 1, no. 1, pp. 5-17, 1998.

[27] P. M. Herr, F. R. Kardes, and J. Kim, "Effects of word-ofmouth and product-attribute information on persuasion: an accessibility-diagnosticity perspective," Journal of Consumer Research, vol. 17, no. 4, pp. 454-462, 1991.

[28] F. T. Su, Investigating Consumers Acceptance Behavior on Tablet PC by an Integrated Model of TAM and TTF, p. 67, National Taipei University of Technology, Taipei City, Taiwan, 2013.

[29] J. I. E. Liao, "A study of the technology acceptance model on sports lottery customers online betting behavior," Degree thesis, p. 141, Chaoyang University of Technology, Taipei City, Taiwan, 2016.

[30] M.-J. Cheng, H.-H. Tsai, S.-W. Hung, and P.-W. Chen, "Exploring the adoption intention through decomposed theory of planned behavior: an empirical study on mobile applications," in Proceedings of the Portland International Conference on Management of Engineering and Technology (PICMET), August 2015.

[31] G. Torkzadeh, X. Koufteros, and K. Pflughoeft, "Confirmatory analysis of computer self-efficacy," Structural Equation Modeling: A Multidisciplinary Journal, vol. 10, no. 2, pp. 263-275, 2003.

[32] K. A. Bollen and R. A. Stine, "Bootstrapping goodness-of-fit measures in structural equation models," Sociological Methods \& Research, vol. 21, no. 2, pp. 205-229, 1992.

[33] J. H. Cheong and M. C. Park, "Mobile internet acceptance in korea," Internet Research, vol. 15, no. 2, 2005.

[34] C. H. Lin, Using Extended Theory of Planned Behavior to Investigate Consumers' Intention of Self-Service Travel Sites, p. 89, Tatung University, Taipei, Taiwan, 2015.

[35] S. A. Rause, "Wearable technology: improving exercise habits and experiences in adults," These and Dissertations, Indiana University of Pennsylvania, Indiana, PA, USA, 2016.

[36] C.-W. Lin, S.-S. Lee, K.-Y. Tang, Y.-X. Kang, C.-C. Lin, and Y.-S. Lin, "Exploring the users behavior intention on mobile payment by using TAM and IRT," in Proceedings of the $3 r d$ International Conference on E-Society, E-Education and E-Technology, Taipei City, Taiwan, August 2019. 